\title{
Anterior cruciate ligament reconstruction using an anterior cruciate ligament stump
}

\author{
Tie-Zhu Chen ${ }^{1,2}$, Yi-Sheng Wang ${ }^{2}$, Xiao-Sheng Li $^{1}$ \\ ${ }^{1}$ Department of Orthopedics, Hunan Provincial People's Hospital, Changsha, China \\ ${ }^{2}$ Department of Orthopedics, the First Affiliated Hospital of Zhengzhou University, Zhengzhou, China
}

Videosurgery Miniinv 2019; 14 (3): 461-467

DOI: https://doi.org/10.5114/wiitm.2019.81305

\begin{abstract}
Introduction: Anterior cruciate ligament (ACL) injury always leads to knee pain and dysfunction for which surgical reconstruction is recommended, with good clinical results, but decreased postoperative proprioception also tends to occur. ACL stump (ACLS)-retaining ACL reconstruction and non-ACLS-retaining ACL reconstruction are the two surgical options.

Aim: To investigate the efficacy of retaining the ACLS in allograft reconstruction.

Material and methods: Thirty patients were retrospectively assigned to group A, ACLS-retaining ACL reconstruction; and group B, non-ACLS-retaining ACL reconstruction, and their data were analyzed. The knee function (Lysholm score and Tegner motion score) and proprioceptive function of the two groups were assessed and compared by postoperative reconstruction angle.

Results: The 30 patients were followed up for a mean 20 months. The mean Lysholm score in group A increased from $55.7 \pm 11.6$ points preoperatively to $95.2 \pm 5.7$ points postoperatively; that in group $B$ increased from $56.7 \pm 11.3$ points preoperatively to $94.6 \pm 7.2$ points postoperatively. The mean Tegner motion score in group $A$ was increased from $2.4 \pm 0.7$ points preoperatively to $6.0 \pm 0.7$ points postoperatively; that in group $B$ increased from $2.73 \pm 0.96$ points preoperatively to $6.24 \pm 0.48$ points postoperatively; the postoperative scores did not differ significantly between the two groups. The proprioception was better in group $A$ than in group $B$ at 3, 6, and 12 months postoperatively $(p<0.05)$.

Conclusions: ACLS-retaining ACL reconstruction has good efficacy and the retained ACLS can benefit postoperative proprioception recovery at an early stage.
\end{abstract}

Key words: anterior cruciate ligament, arthroscopy, allograft ligament, proprioception, stump reservation.

\section{Introduction}

Anterior cruciate ligament $(A C L)$ is the main structure that maintains knee stability, and $A C L$ injury is a common clinical disease with arthroscopy as the main method of treating $A C L$ injuries. The $A C L$ injuries can produce significant dorsal instability of the knee joints, meniscal tear, or articular cartilage degeneration, leading to knee pain and dysfunction.
Numerous studies have demonstrated that conservative $A C L$ injury treatment failure rates are as high as $50-60 \%$, whereas surgical reconstruction can achieve good clinical results in $80-90 \%$ of cases [1]. Functional studies of knee joint proprioception have increased in recent years. In 1984, Schultz first described in detail the ACL mechanoreceptors and divided them into four categories: Ruffini corpuscles, Pacinian corpuscles, Golgi tendon organs, and free

\section{Address for correspondence}

Xiao-Sheng Li, Department of Orthopedics, Hunan Provincial People’s Hospital, 61 Jiefang West Road, 410000 Changsha, China, phone: +8673183929222 , e-mail: xiaoshenglidoc@126.com 
nerve endings. Schutte pointed out that neural components form $1-2.5 \%$ of the total $A C L$ volume and that most are distributed beneath the synovium of the bilateral ligament ends [2]. Johansson found that the nerves dominating the $A C L$ are the posterolateral articular nerves originating from the posterior tibial nerve [3]. Knee cruciate ligament repair involves restoring the stable structure and kinetic stability as well as the proprioception and nerves. Friden measured the motion perception threshold of 16 patients with acute $A C L$ injury in the lateral position, among whom 4 exhibited related continuous significant proprioceptive loss in post-injury months 1,2 , and 8 to those combining cartilage and meniscus injuries [4]. Roberts measured the motion perception threshold of 54 patients with $A C L$ injury in their lateral position and found that decreased proprioception after ACL injury is related to cartilage damage, joint laxity, and age [5]. The meta-analysis performed by Relph revealed that the knee proprioception of patients with $A C L$ injuries is significantly weaker than that of non-injured individuals, while the proprioception of the injured knee is weaker than that of the non-injured side and the proprioception after repair is better than that before [6].

To study the impact of retaining the ACL Stump (ACLS) on postoperative knee function and proprioception, this study randomly divided 30 patients into 2 groups $(n=15)$. Between the ACLS-retaining group (group A), the ACL injury patients whose anterior cruciate ligament of tibial residue was preserved with allograft for $A C L$ reconstruction, and the non-ACLS-retaining group (group $B$ ), the $A C L$ injury patients whose anterior cruciate ligament of tibial residue was not preserved with allograft for $A C L$ reconstruction, a comparison of their treatment efficacies was conducted.

\section{Aim}

The aim of this study was to investigate the efficacy of retaining the ACLS in ACL allograft reconstruction.

\section{Material and methods}

\section{Case information}

A total of 30 patients with ACL injuries who underwent allogeneic tendon fixation using absorbable screws (Beijing Yunkang Co.) at Hunan Provincial People's Hospital between July 2010 and December 2011 were collected and randomly divided into two groups: group $A$ and group $B$. In group $A(n=15 ; 8$ males, 7 females; mean age, 28.4 years (range: $17-48$ years)), ACLS-retaining ACL reconstruction was performed to correct a left knee injury in 10 patients and right knee injury in 5 patients. The main symptoms included knee pain, limping, interlocking, knee instability when going up and down stairs, or squatting difficulty, the mean knee Lysholm score was $58.5 \pm 12.5$ points and the mean Tegner score was $2.44 \pm 0.71$ points.

In group $B(n=15 ; 9$ males, 6 females; mean age, 27.6 years (range: $16-44$ years)), non-ACLS-retaining $A C L$ reconstruction was performed to correct a left knee injury in 8 patients and a right knee injury in 7 patients. The mean knee Lysholm score [7] was $56.7 \pm 11.3$ points, while the mean Tegner score was $2.73 \pm 0.96$ points. This study was conducted in accordance with the Declaration of Helsinki. Written informed consent was obtained from all participants.

\section{Conventional microscopy}

All procedures were completed by the same group of physicians. After the induction of epidural anesthesia, each patient was inspected for injuries to the synovium, cartilage, ligaments, and meniscus starting from the anterior patellar bursa with the anterolateral approach as the observation approach and the anterointerior approach as the operating approach. Once the ACL injury situation was clear, the allogenic tendon was soaked in $500 \mathrm{ml}$ of normal saline plus $10 \mathrm{mg}$ of dexamethasone for $30 \mathrm{~min}$. The ligament was then prepared into segments $12-15 \mathrm{~cm}$ long and 8-9 $\mathrm{mm}$ in diameter, which were then braided into one bunch with a 3/0 Vicryl suture. Both ends were drilled by 5-0 Ethibond suture to suture the ligament ends in the same way to provide traction with $60 \mathrm{~N}$ of force for $8 \mathrm{~min}$, then the intra-articular synovial and meniscal injuries were cured.

\section{Establishing the tibiofemoral tunnel}

The main difference between the two groups was the way to treat the anterior cruciate ligament of tibial residue. In group $A$, the $A C L$ femoral end and middle injured fiber were eliminated with retention of about $1 \mathrm{~cm}$ of fiber at the ligament tibial end point; in group $B$, all of the fibrous tissue of the $A C L$ was removed. The footprint positioning method was then used (55 angle; ACL Locator; Smith-Nephew Co.) with the tunnel entrance setting at the center of the orig- 
inal ACL tibial stump, the guide arm being parallel to the tibial plateau and the guide pin being parallel to the direction of the original ACL fibers. Special attention should be paid to protecting the ligament edge stump tissue to avoid damaging the femoral condyle. According to the graft diameter, one tibial tunnel drill of the same size was used to expand the tibial tunnel along the guide pin (retaining the cancellous bone on the drill); after the tunnel was established, the sharp edges of the tunnel were removed using one curette. One femoral guider (ligament diameter/2 + $2 \mathrm{~mm}$ in size) was then implanted via the anteromedial approach, positioned at the intercondylar fossa (10:30 [right knee)/1:30 [left knee]), and fixed to the posterior wall of the femoral condyle to ensure the integrity of the posterior bone cortex of the femoral intercondylar fossa. The guide needle was then drilled in and pierced out of the skin; another femoral drill with the same diameter as the ligament was used to prepare the femoral tunnel about $3-3.5 \mathrm{~cm}$ in length with the femoral guide retained inside the tunnel. The sharp edges of the tunnel were removed using one curette.

\section{Ligament installation}

The ligament allograft was induced into the joint cavity via the tibial tunnel, the reconstructed ligament was connected with the anterior cruciate ligament of tibial residue in the middle without any impact in the active joint cavity followed by flexing of the knee to $120^{\circ}$, insertion of the guide needle of the absorbable interface screws via the anteromedial approach, insertion of absorbable interface screws of the same diameter (Smith \& Nephew), and fixing of the femoral side of the ligament allograft. The tibial traction line was then tractioned to flex the knee 20 times to adjust the ligament tension. Thereafter, absorbable interface screws (Smith \& Nephew) were used to fix the tibial side of the graft under tension to maintain $30^{\circ}$ of knee flexion. After the surgery, elastic bandages were wrapped from the heel to the mid-thigh together with a movable brace to maintain the extended position.

\section{Postoperative treatment}

All patients underwent postoperative preventive anti-immune therapy, namely dexamethasone $20 \mathrm{mg}$ $+100 \mathrm{ml}$ of saline intravenous glucose tolerance test (ivGTT) on postoperative days 1 and 2, dexamethasone $10 \mathrm{mg}+100 \mathrm{ml}$ of saline ivGTT on postoperative days 3 and 4, and dexamethasone $5 \mathrm{mg}+$
$100 \mathrm{ml}$ of saline ivGTT + $20 \mathrm{mg}$ tripterygium glycoside tablets tid on postoperative day 5 , and $20 \mathrm{mg}$ tripterygium glycoside tablets tid starting on postoperative day 6 for 3 months. Cefuroxime $2 \mathrm{~g}$ ivGTT was used $30 \mathrm{~min}$ before surgery, and cefuroxime $2 \mathrm{~g}$ q8h was also applied after surgery to prevent infection. Quadriceps exercises and ankle pump exercises were started in the early postoperative stage to exercise the isometric contraction of the quadriceps and gastrocnemius under movable brace protection. The brace angle was adjusted to $30^{\circ} 3$ days after surgery and gradually increased until $90^{\circ}$ was achieved within 2 weeks and $120^{\circ}$ was achieved within 6 weeks, followed by 3-month brace protection. Non-weightbearing walking with crutches was allowed 5 weeks after surgery and full-weight-bearing ambulation was allowed 12 weeks later. Jogging and non-confrontational training were allowed 6 months later, while sports were allowed 1 year after surgery.

\section{Follow-up and evaluation}

All patients were followed up for a mean 20 months (range: 16-24 months). X-ray images of the normal and lateral sides of the knee taken before and immediately after surgery were compared to monitor for graft fixation failure, bone tunnel enlargement, intercondylar fossa impingement, or knee instability. The results of the anterior drawer test, Lachman signs, and knee Lysholm and Tegner scores before versus after surgery were compared to evaluate the function of the diseased limb.

All patients were followed up at months 3, 6, and 12 , and the angle reconstruction method was used to measure the differences between the reconstructed and preset angles with knee flexion at $0^{\circ}, 30^{\circ}$, and $45^{\circ}$, respectively. The knee of each patient was passively set at $0^{\circ}, 30^{\circ}$, and $45^{\circ}$, respectively, using a continuous passive motion machine; after $5 \mathrm{~s}$, it was set at the maximum extension position and the patient was given $15 \mathrm{~s}$ to reproduce the preset angle to obtain the difference between the reconstructed and preset angles. The mean of 5 measurements was used to represent the position sense of the knee joint.

\section{Statistical analysis}

SPSS 18.0 statistical software was used for the analyses, and Lysholm scores, Tegner scores, and angle reconstruction methods before versus after ligament reconstruction were compared using the 
paired $t$ test with the test level set at $\alpha=0.05$ and values of $p<0.05$ considered statistically significant.

\section{Results}

All of the patients returned to the hospital for a mean follow-up time of 20 months (range: 16-24 months); X-ray and MRI images were taken of the normal and lateral knee sides and the results revealed that the graft was firmly fixed, the bone tunnel did not expand, no intercondylar fossa impingement was found, no cyclopia had formed, and the symptoms of knee instability had disappeared.

The mean post-operative Lysholm score (Table I) and Tegner motor function score (Table II) were higher than the preoperative score. The intergroup comparison of angle reconstruction method at 3 months and 6 months showed a significant difference but not at 12 months (Table III).

\section{Discussion}

\section{Efficacy of ligament allograft for reconstructing $\mathrm{ACL}$}

This method can avoid complications of ligament autografts such as patellar fracture, patellar tendon rupture, anterior knee pain, and quadriceps weak- ness or saphenous nerve injury after the knee extensor is damaged; in particular, it increases the advantages of medial knee instability when combined with medial knee instability. Sun et al. [7] performed a mean 42.2-month follow-up to test the functions, subjective evaluation, and motion levels between patients who underwent autologous hamstring allografting for $\mathrm{ACL}$ reconstruction and those who underwent ligament allografting and found no statistically significant intergroup difference. This study used ligament allografts to reconstruct the $\mathrm{ACL}$, and the postoperative follow-up revealed that the patients' knee instability had disappeared, the anterior drawer test and Lachman test results improved significantly, and the Lysholm and Tegner scores improved significantly, proving that the treatment is effective. The 20-month follow-up (range: 16-24 months) achieved a $100 \%$ excellent rate, consistent with previous reports. One patient in this study exhibited a yellow thin exudate outside the mouth of the tibial tunnel 3 days after surgery with the floating patella test $( \pm)$, knee swelling, and local knee skin temperature increased compared to the contralateral limb, but the bloodwork was still within the normal range. The C-reactive protein level and erythrocyte sedimentation rate, considered signs of al-

Table I. Lysholm scores

\begin{tabular}{|lcc|}
\hline Variable & Group A $(n=15)$ & Group B $(n=15)$ \\
\hline Before surgery & $55.7 \pm 11.6$ & $56.7 \pm 11.3$ \\
\hline 20 months after surgery & $95.2 \pm 5.7$ & $94.6 \pm 7.2$ \\
\hline & $t=-13.6, p<0.05$ & $t=-15.2, p<0.05$ \\
\hline
\end{tabular}

Table II. Tegner motion scores

\begin{tabular}{|lcc|}
\hline Variable & Group A $(n=15)$ & Group B $(n=15)$ \\
\hline Before surgery & $2.4 \pm 0.7$ & $2.73 \pm 0.96$ \\
\hline 20 months after surgery & $6.0 \pm 0.7$ & $6.24 \pm 0.48$ \\
\hline & $t=-26.8, p<0.05$ & $t=-22.1, p<0.05$ \\
\hline
\end{tabular}

Table III. Results of angle reconstruction method

\begin{tabular}{|c|c|c|c|c|c|c|c|c|c|c|}
\hline \multirow[t]{2}{*}{ Group } & \multirow[t]{2}{*}{$N$} & \multicolumn{3}{|c|}{3 months after surgery } & \multicolumn{3}{|c|}{6 months after surgery } & \multicolumn{3}{|c|}{12 months after surgery } \\
\hline & & $45^{\circ}$ & $30^{\circ}$ & $0^{\circ}$ & $45^{\circ}$ & $30^{\circ}$ & $0^{\circ}$ & $45^{\circ}$ & $30^{\circ}$ & $0^{\circ}$ \\
\hline A & 15 & $3.52 \pm 0.72$ & $3.43 \pm 0.85$ & $2.13 \pm 0.49$ & $3.01 \pm 0.91$ & $2.47 \pm 0.66$ & $2.06 \pm 0.74$ & $2.84 \pm 0.63$ & $2.55 \pm 0.51$ & $2.02 \pm 0.48$ \\
\hline B & 15 & $6.78 \pm 1.35$ & $5.84 \pm 1.23$ & $3.52 \pm 0.88$ & $4.98 \pm 1.46$ & $4.87 \pm 1.02$ & $3.27 \pm 0.92$ & $4.52 \pm 0.77$ & $4.26 \pm 0.65$ & $3.15 \pm 0.68$ \\
\hline$P$-value & & $<0.05$ & $<0.05$ & $<0.05$ & $<0.05$ & $<0.05$ & $<0.05$ & $<0.05$ & $<0.05$ & $<0.05$ \\
\hline
\end{tabular}


lograft tissue rejection (not excluded infection), were slightly increased. This patient was strengthened by re-dressing; we adequately drained the exudate from the tibial tunnel mouth by removing the stitches and prolonged the injection time of dexamethasone. On postoperative day 7 , no significant exudate was visible from the wound, and this patient was withdrawn from the dexamethasone injections but administered tripterygium glycosides $20 \mathrm{mg}$ tid to confront the rejection. This patient exhibited good wound healing. Cryopreservation effectively reduces the antigenicity of allogenic ligaments, so the tissue rejection rate is rarely low; meanwhile, we applied dexamethasone injection and tripterygium glycosides routinely after surgery to confront the rejection and found no cases of serious rejection reactions.

\section{ACLS-retaining $A C L$ reconstruction}

\section{ACLS-retaining}

During $\mathrm{ACL}$ reconstruction surgery, cleaning the stump can guarantee a good surgical field that is ample and conducive to graft positioning and fixation; meanwhile, the procedure itself is relatively simple and can avoid intercondylar fossa impingement, ensure the femoral tunnel position and posterior wall thickness precision, and reduce the incidence of cyclopia. However, clearing ACLS is not conducive to reconstructing the graft's blood supply, and clearing the original ligament's mechanoreceptors negatively impacts knee stability and neuromuscular reflexes. Clinically, ACL injury sites are mainly located near the femoral end, so ACLS at the tibial end point is relatively more complete, forming a solid foundation to preserve the $\mathrm{ACL}$ tibial stump.

After ACL reconstruction, the graft has no blood supply, so necrosis and revascularization will likely occur. Blood supply reconstruction mainly relies on the ingrowing of blood vessels from the surrounding synovium and fat pad, so careful handling of the fat pad is needed to facilitate graft vascularization. Gohil et al. [8] cleaned the intercondylar fossa of the 25 of 49 patients who underwent $A C L$ reconstruction and retained the ACLS in 24 patients. The MRI performed 2, 6, and 12 months later revealed that revascularization in the $A C L$ reconstruction grafts with a fiber stump was established earlier than in those without. Is $\mathrm{ACL}$ reconstruction with tibial stump retaining more prone to lead to inaccurate bone tunnel positioning and intercondylar fossa impingement syndrome? Wang et al. found [9] 45 cases of cyclopia, among which the removed fibrous nodules exhibited disordered fibrous connective tissue under the microscope. Partial samples contained cartilage-like tissue, so cyclopia is formed by the calcification of deposited para-tunnel cartilage and smashed bone. However, Cha et al. [10] compared the incidence of cyclopia between stump-retaining $\mathrm{ACL}$ reconstruction and non-stump-retaining $A C L$ reconstruction by $M R I$, found that they were similar, and concluded that cyclopia is not related to ACLS retention. Gohil et al. [11] found that cyclopia usually appeared 6-12 months after ACL reconstruction. The incidence of cyclopia between stump-retaining and non-stump-retaining $\mathrm{ACL}$ reconstruction did not differ significantly, with a mean rate of $46.8 \%$; retaining the stump does not lead to an increased risk of cyclopia. Lee et al. [12] considered that the retained stump covered the tunnel exit, so the bone chips within the tibial tunnel cannot be exposed to the joint cavity, so it can reduce bone formation in front of the graft and reduce cyclopia. In the ACLS-retaining group, the tibial end of the $A C L$ was retained about $10 \mathrm{~mm}$, and the femoral tunnel was located by the anteromedial approach using the femoral offset guide. When drilling the femoral tunnel, care should be taken that the residual tibial end of the $A C L$ is not entangled on the drill bit. In group $A$, the reconstructed ligament tissue penetrated from the center of the residual tibial end, and PDS II suture was used to avoid the sagging of the end. In group B, the residual tibial end of the anterior cruciate ligament was completely removed. No impact of the intercondylar fossa or cyclopia was found in the two groups during a 20-month follow-up (16-24 months). The post-operative Lysholm score and Tegner motor function score of the two groups were better than the pre-operative scores. Anterior cruciate ligament reconstruction with allograft can achieve good postoperative stability and satisfactory motor function recovery.

\section{Proprioception of $\mathrm{ACL}$}

The ACL has become a hot topic for acting as the nerve organ that is involved in and constitutes knee proprioception. $\mathrm{ACL}$ mechanoreceptors are divided into four categories, account for $1-2.5 \%$ of the total $A C L$ volume, are mainly distributed inside the synovium of bilateral ligaments, and are dominated 
by the posterior tibial nerve. In 1994, Denti et al. [13] studied the changes of mechanoreceptors after ACL injury and found under no special treatment, the mechanoreceptors 3 months after ACL injury are basically normal but their quantity is slightly reduced; 9 months later, only a small amount of free nerve endings are left; 1 year later, all of the mechanoreceptors have disappeared. Dhillon et al. [14] found via histological observation that the longer the $A C L$ injury time is, the more serious is the degeneration of stump mechanoreceptors and proprioceptors. Statistically significant differences have been found between injury time and injury degree of the mechanical sensory fibers; it is believed that ACLS retention can potentially benefit the postoperative recovery of proprioception. Georgoulis et al. [15] found the existence of mechanoreceptors attaching to the posterior ACL stump 3 years after injury. Relph found via a meta-analysis that the knee proprioception of patients with an ACL injury is significantly weaker than that in non-injured individuals, the proprioception of the injured knee is weaker than that of the non-injured side, and the proprioception is better after than before surgery [6]. Authors found that ACL reconstruction with stump retention can contribute to knee function and proprioception recovery and exhibit certain protective effects toward the reconstructed ligaments [16]. Lee et al. performed ACLS-retaining ACL reconstruction in 16 patients followed for a mean of 35.1 months and found better proprioception than that of non-ACLS-retaining $\mathrm{ACL}$ reconstruction [12]. More doctors have performed related studies, and their follow-up data also proved that retaining the stump can certainly contribute to postoperative proprioception recovery $[14,17,18]$. Merter used the Cybex apparatus to measure and compare the proprioception after allogeneic and autologous $A C L$ reconstruction, and the results showed no difference in postoperative proprioception [19]. Noh covered the graft surface with the retained $A C L$ stump, which finally improved the healing speed of the graft, increased its strength, and improved knee joint proprioception [20]. In this study, the angle reconstruction method revealed a significant difference at 3 months and 6 months but no difference was found at 12 months, which meant that in group $A$, the proprioception of $A C L$ was better than in group $B$. The reason may be that there were a large number of mechanoreceptors in the residual tibial end of the anterior cruciate ligament, which was related to the proprioception of the knee joint. The preservation of the tibial end retains part of the anterior cruciate ligament, which is beneficial to the recovery of the proprioceptive function of the knee joint in the early stage. However, there is no significant difference between the two groups after 12 months. The reason may be partial inactivation of mechanical receptors and compensation of proprioceptive functions from other parts of the knee joint.

\section{Conclusions}

This study retained the ACL stump in cases of allogenic $A C L$ reconstruction, and our results revealed that the postoperative function scores were improved after compared to before, as described in previous reports, which may be related to the fact that the retained stump can benefit from the vascular ingrowth of the graft and promote autologous ligament reconstruction. Furthermore, retaining the stump can reduce the loss of nerve receptors, thus helping restore the proprioception and better restore knee motion and sensory function. Thus, ACLS-retaining $A C L$ reconstruction can achieve satisfactory clinical results at the early stage.

\section{Conflict of interest}

The authors declare no conflict of interest.

\section{References}

1. Fu FH, Bennett CH, Ma CB, et al. Current trends in anterior cruciate ligament reconstruction. Part II. Operative procedures and clinical correlations. Am J Sports Med 2000; 28: 124-30.

2. Schutte MJ, Dabezies EJ, Zimny ML, et al. Neural anatomy of the human anterior ligament. Bone Joint Surg Am 1987; 69: 243-7.

3. Johansson $H$, Sjolander P, Sojka P. A sensory role for the cruciate ligament. Clin Orthop Relat Res 1991; 268: 161-78.

4. Fridèn T, Roberts D, Zätterström R, et al. Proprioceptive defects after an anterior cruciate ligament rupture-the relation to associated anatomical lesions and subjective knee function. Knee Surg Sports Traumatol Arthrosc 1999; 7: 226-31.

5. Roberts D, Andersson G, Fridén T. Knee joint proprioception in ACL-deficient knees is related to cartilage injury, laxity and age: a retrospective study of 54 patients. Acta Orthop Scand 2004; 75: 78-83.

6. Relph N, Herrington L, Tyson S. The effects of ACL injury on knee proprioception: a meta-analysis. Physiotherapy 2014; 100: 18795.

7. Sun K, Zhang J, Wang Y, et al. Arthroscopic anterior cruciate ligament reconstruction with at least 2.5 years' follow-up comparing hamstring tendon autograft and irradiated allograft. Arthroscopy 2011; 27: 195-202. 
8. Gohil S, Annear PO, Breidahl W. Anterior cruciate ligament reconstruction using autologous double hamstrings: a comparison of standard versus minimal debridement techniques using MRI to assess revascularisation. A randomised prospective study with a one-year follow-up. J Bone Joint Surg Br 2007; 89: 1165-71.

9. Wang J, Ao Y. Analysis of different kinds of cyclops lesions with or without extension loss. Arthroscopy 2009; 25: 626-31.

10. Cha J, Choi SH, Kwon JW, et al. Analysis of cyclops lesions after different anterior cruciate ligament reconstructions: a comparison of the single bundle and remnant bundle preservation techniques. Skeletal Radiol 2012; 41: 997-1002.

11. Gohil S, Falconer TM, Breidahl W, et al. Serial MRI and clinical assessment of cyclops lesions. Knee Surg Sports Traumatol Arthrosc 2014; 22: 1090-6.

12. Lee BI, Kwon SW, Kim JB, et al. Comparison of clinical results according to amount of preserved remnant in arthroscopic anterior cruciate ligament reconstruction using quadrupled hamstring graft. Arthroscopy 2008; 24: 560-8.

13. Denti M, Monteleone M, Berardi A, et al. Anterior cruciate ligament mechanoreceptors. Histologic studies on lesions and reconstruction. J Clin Orthop Relat Res 1994; 308: 29-32.

14. Dhillon MS, Bali K, Vasistha RK. Immunohistological evaluation of proprioceptive potential of the residual stump of injured anterior cruciate ligaments (ACL). J Int Orthop 2010; 34: 737-41.

15. Georgoulis AD, Pappa L, Moebius U, et al. The presence of proprioceptive mechanoreceptors in the remnants of the ruptured $A C L$ as a possible source of re-innervation of the $A C L$ autograft. Knee Surg Sports Traumatol Arthrosc 2001; 9: 364-8.

16. Takazawa $\mathrm{Y}$, Ikeda $\mathrm{H}$, Kawasaki $\mathrm{T}$, et al. ACL reconstruction preserving the ACL remnant achieves good clinical outcomes and can reduce subsequent graft rupture. Orthop J Sports Med 2013; 1: 2325967113505076.

17. Dhillon MS, Bali K, Prabhakar S. Proprioception in anterior cruciate ligament deficient knees and its relevance in anterior cruciate ligament reconstruction. Indian J Orthop 2011; 45: 294-300.

18. Kim SJ, Jo SB, Kim TW, et al. A modified arthroscopic anterior cruciate ligament double-bundle reconstruction technique with autogenous quadriceps tendon graft: remnant-preserving technique. Arch Orthop Trauma Surg 2009; 129: 403-7.

19. Ozenci AM, Inanmaz E, Ozcanli H, et al. Proprioceptive comparison of allograft and autograft anterior cruciate ligament reconstructions. Knee Surg Sports Traumatol Arthrosc 2007; 15: 1432-7.

20. Noh JH, Yoon KH, Song SJ, et al. Re-tensioning technique to cover the graft with remnant in anterior cruciate ligament reconstruction. Arthrosc Tech 2014; 3: e679-82.

Received: 8.09.2018, accepted: 19.11.2018. 